LTH 1077

\title{
Bilinear quark operator renormalization at generalized symmetric point
}

\author{
J.M. Bell \& J.A. Gracey, \\ Theoretical Physics Division, \\ Department of Mathematical Sciences, \\ University of Liverpool, \\ P.O. Box 147, \\ Liverpool, \\ L69 3BX, \\ United Kingdom.
}

\begin{abstract}
We compute Green's functions with a bilinear quark operator inserted at non-zero momentum for a generalized momentum configuration to two loops. These are required to assist lattice gauge theory measurements of the same quantity in matching to the high energy behaviour. The flavour non-singlet operators considered are the scalar, vector and tensor currents as well as the second moment of the twist-2 Wilson operator used in deep inelastic scattering for the measurement of nucleon structure functions.
\end{abstract}




\section{Introduction.}

The protons and neutrons of the atomic nucleus are known nowadays to be comprised of fundamental point-like particles called quarks. These are held in the nucleon bound states by the strong colour force whose quanta are gluons. Neither quarks nor gluons have been seen in isolation in nature due to confinement, which is sometimes called infrared slavery. This term originates in part from the fact that when protons and neutrons are probed experimentally at high energies they behave as free fundamental entities in that asymptotic regime. This manifestation as apparently free particles is an apparition since it is not the picture at all energy scales. At low energies the colour glue restricts the release of isolated quarks. While high energy experiments have demonstrated that our theoretical understanding at a Lagrangian level is essentially correct, via the non-abelian gauge theory known as Quantum Chromodyanmics (QCD), how protons and neutrons actually form and become the observed degrees of freedom is still not fully resolved. One way of studying properties of the nucleon substructure is to model the internal quark behaviour numerically. This goes well beyond the perturbative region of QCD and requires the technique known as lattice gauge theory. The proton and neutron structure functions can be constructed from models of the non-perturbative behaviour with lattice gauge theory input data and in the main this has progressed our understanding of nucleon structure.

Given this overview there is a technical side to actually extracting the information required for the nucleon structure functions. Based on the operator product expansion one computes moments of the structure functions which are related to Green's functions involving twist-2 operators, [1. For the flavour non-singlet sector of the structure functions the $n$th moment of these operators are quark anti-quark operators involving $(n-1)$ covariant derivatives. Their renormalization properties have been known for many years with the provision of the one, two and three loop $\overline{\mathrm{MS}}$ anomalous dimensions, 1, 2, 3. However, the dependence of the Green's function itself on momenta and the strong coupling constant is what is necessary for proton and neutron structure analysis. As noted this is the quantity computed on the lattice nonperturbatively. However, these calculations are intensely numerical and the measurement of a central value at a particular momentum is only part of the work. The second aspect is to refine the error bounds so that they are small. One aspect of that analysis is that in addition the behaviour of the measured Green's function should match onto the high energy piece. The latter is determined in perturbation theory, [1, 2, 3]. Indeed this has been the modus operandi for the last decade or so. Multiloop evaluation of the same Green's function can be determined to two and three loops. For the most part the latter order is for the momentum configuration where the operator is at zero momentum insertion in a quark 2-point function. However, more recently the focus has switched to forward matrix elements which requires a non-zero momentum flowing through the inserted operator. This relates to generalized parton distribution functions.

In this instance the supporting perturbative computations have been carried out at the fully symmetric momentum configuration but only to two loops. Such results, [4, 5, 6, 7, 8, have proved important in lattice analyses. For example, see [9, 10, 11, 12, 13, 14, 15, 16, 17, 18, 19, 20, $21,22,23,24,25,26,27,28,29,30$ ] for a non-exhaustive representation. While this has provided a useful start for such forward matrix element analyses, it is slightly restrictive. In computing at the fully symmetric point the squares of all incoming external momenta are equal, [4, 5, 17, 8]. That value for the operator insertion is no different from that of the external quark legs on the Green's function. Instead a more general configuration would be preferable where the momenta flowing into the operator itself is not tied to those of the other legs. Therefore, it is the purpose of this article to address this problem. We will compute the Green's function of the relevant quark operators, which are twist-2 flavour non-singlet operators. The particular momentum configuration considered will be called the interpolating momentum (IMOM) subtraction point. 
This is in contradistinction to the symmetric momentum (SMOM) subtraction point of [4, 5, 7, 8]. The origin of our IMOM nomenclature is due to the appearance of a parameter, $\omega$, which will be free. It parametrizes the momentum transfer at the operator insertion. Our results will all be functions of $\omega$ and will provide a freedom for the lattice to tune their analysis. For instance, it may transpire that for certain values of $\omega$ the error bounds are tighter than for others. Our choice of IMOM configuration, which is a non-exceptional one, is not original in that it was considered in [6] for the renormalization of the quark mass operator. As a first stage we will reconstruct that computation [6] partly as a check on our computational setup but also because we will give the full decomposition of the Green's function into its Lorentz basis. We repeat the process for both the vector current and tensor quark bilinear. The former is considered because of its later relation to the second moment of the twist-2 flavour non-singlet operator of deep inelastic scattering. The tensor operator is evaluated because of its relation to the vector meson decay constant, [31, for instance. Our analysis is completed by the calculation which is that of the full set of second moment twist-2 operators. With the increase in Lorentz indices in the case of this set of operators, the basis of tensors for the Green's function itself enlarges. However, we will compute the full result. This is necessary as lattice regularized QCD makes measurements in different directions in order to decipher the signal which contributes to the matrix element for the structure function. Throughout we work in the $\overline{\mathrm{MS}}$ scheme and do not consider any of the hybrid renormalization schemes which were introduced in earlier work such as the modified regularization invariant $\left(\mathrm{RI}^{\prime}\right)$, [32, 33], or momentum subtraction (MOM) schemes, [34, 35]. This is partly as lattice motivated schemes can readily be converted to the reference $\overline{\mathrm{MS}}$ scheme but also because there is a large degree of freedom in defining a non-minimal momentum subtraction scheme which derives from RI'.

The paper is organized as follows. The definition of the operators we consider and the quantum field theoretic formalism used is introduced in section 2. This includes a discussion on the technical details of the computation. The subsequent two sections contain the main results with section 3 concentrating on the basic bilinear quark operators while section 4 focuses on the twist-2 Wilson operator second moment. We provide concluding remarks in section 5 . An appendix records the tensor basis for the Green's function of each operator together with the explicit projection matrices which are $\omega$-dependent. The latter are required to extract the coefficient of each basis tensor as a function of $\omega$.

\section{Background.}

We begin by briefly reviewing the formalism we use which will be based on earlier articles, [5, 6, 7, 8]. We focus on the main differences due to the more general external momentum configuration. We refer the interested reader to these articles for more detail but our notation will be the same as [7, 8]. First, for shorthand and for labelling purposes the operators we consider are

$$
S \equiv \bar{\psi} \psi, V \equiv \bar{\psi} \gamma^{\mu} \psi, T \equiv \bar{\psi} \sigma^{\mu \nu} \psi, \quad W_{2} \equiv \mathcal{S} \bar{\psi} \gamma^{\mu} D^{\nu} \psi \quad, \quad \partial W_{2} \equiv \mathcal{S} \partial^{\mu}\left(\bar{\psi} \gamma^{\nu} \psi\right)
$$

where $\sigma^{\mu \nu}=\frac{1}{2}\left[\gamma^{\mu}, \gamma^{\nu}\right]$. The letters $S, V, T, W_{2}$ and $\partial W_{2}$ denote scalar, vector, tensor, twist-2 flavour non-singlet second moment Wilson operator and total derivative of the latter respectively. In the case of $W_{2}$ and $\partial W_{2}$ each operator is symmetrized with respect to the Lorentz indices and is also traceless in $d$-dimensions. The operator for this latter procedure is denoted by $\mathcal{S}$ and if

$$
\mathcal{O}_{\mu \nu}^{W_{2}}=\bar{\psi} \gamma_{\mu} D_{\nu} \psi
$$


for instance, then, [1, 2],

$$
\mathcal{S} \mathcal{O}_{\mu \nu}^{W_{2}}=\mathcal{O}_{\mu \nu}^{W_{2}}+\mathcal{O}_{\nu \mu}^{W_{2}}-\frac{2}{d} \eta_{\mu \nu} \mathcal{O}_{\sigma}^{W_{2} \sigma}
$$

in $d$-dimensions. For each of the operators in (2.1) we will evaluate the Green's function

$$
\left.\left\langle\psi(p) \mathcal{O}_{\mu_{1} \ldots \mu_{n_{L}}}^{L}(r) \bar{\psi}(q)\right\rangle\right|_{\omega}
$$

where $L=S, V, T, W_{2}$ or $\partial W_{2}$ and

$$
p+q+r=0
$$

by conservation of momentum. The restriction here is shorthand notation for the evaluation of the squares of the three external momentum at the interpolating momentum subtraction point we are interested in. In particular the squared momentum flowing into the operator will always be the one which is distinct from the quark leg momenta given that we take, [6],

$$
p^{2}=q^{2}=-\mu^{2}, r^{2}=-\omega \mu^{2}
$$

which imply

$$
p q=\left[1-\frac{\omega}{2}\right] \mu^{2}, \quad p r=q r=\frac{\omega}{2} \mu^{2} .
$$

The $\omega \rightarrow 1$ limit will correspond to the symmetric point used in SMOM. On a note of caution the $\omega \rightarrow 0$ limit will be infrared unsafe and will not be considered in any analysis. Equally the value of $\omega=4$ corresponds to a collinear singularity. Therefore we will only allow $\omega$ to be in the range $0<\omega<4$. The number of free Lorentz indices $n_{L}$ for each operator is indicated in (2.1).

\begin{tabular}{|c||c|c|c|c|}
\hline$L$ & $S$ & $V$ & $T$ & $W_{2}$ \\
\hline$N_{L}$ & 2 & 6 & 8 & 10 \\
\hline
\end{tabular}

Table 1. Number of Lorentz basis elements, $N_{L}$, for each operator insertion $L$.

In order to provide the structure of the Green's function which lattice gauge theory requires for the measurements and matching we decompose (2.4) into its Lorentz basis at the IMOM subtraction point which we will sometimes refer to as an asymmetric point. Specifically we write, [7, 8],

$$
\left.\left\langle\psi(p) \mathcal{O}_{\mu_{1} \ldots \mu_{n_{L}}}^{L}(r) \bar{\psi}(q)\right\rangle\right|_{\omega}=\left.\sum_{k=1}^{N_{L}} \mathcal{P}_{(k) \mu_{1} \ldots \mu_{n_{L}}}^{L}(p, q) \Sigma_{(k)}^{L}(p, q)\right|_{\omega}
$$

where $\left.\Sigma_{(k)}^{L}(p, q)\right|_{\omega}$ are the scalar amplitudes at the IMOM point associated with each respective Lorentz tensor basis element, $\mathcal{P}_{(k) \mu_{1} \ldots \mu_{n}}^{L}(p, q)$. The number of tensors in the basis, $N_{L}$, is given in Table 1 and the basis for each of the operators considered here is given explicitly in Appendix A. To determine the scalar amplitudes we extend the projection method used in previous articles to the specific momentum configuration of interest. In particular the $k$ th amplitude can be extracted by applying the projection matrix $\mathcal{M}_{k l}^{L}$ to the Green's function (2.4) itself. In other words

$$
\left.\Sigma_{(k)}^{L}(p, q)\right|_{\omega}=\left.\mathcal{M}_{k l}^{L} \mathcal{P}_{(l)}^{L}{ }^{\mu_{1} \ldots \mu_{n}}(p, q)\left(\left\langle\psi(p) \mathcal{O}_{\mu_{1} \ldots \mu_{n_{L}}}^{L}(-p-q) \bar{\psi}(q)\right\rangle\right)\right|_{\omega}
$$

where there is a sum over the amplitude label $l$. The projection matrix is deduced from the basis tensors and is defined as the inverse of the matrix given by, [7, 8],

$$
\mathcal{N}_{k l}^{L}=\left.\mathcal{P}_{(k) \mu_{1} \ldots \mu_{n_{L}}}^{L}(p, q) \mathcal{P}_{(l)}^{L \mu_{1} \ldots \mu_{n} L}(p, q)\right|_{\omega} .
$$


The entries in $\mathcal{M}_{k l}^{L}$ and $\mathcal{N}_{k l}^{L}$ are polynomials in $d$ and recorded in the former case for each operator in Appendix A. Due to the asymmetry of the momentum configuration both matrices are $\omega$ dependent. As is evident several elements are singular at $\omega=0$ reflecting the infrared issue at a nullified operator insertion.

Having discussed the quantum field theory formalism underlying the problem we now summarize the practicalities behind determining the amplitudes. We have evaluated all the one and two loop Feynman integrals using an automatic symbolic manipulation programme written in the language Form, [36, 37]. This is the most efficient tool to handle the large amounts of intermediate algebra. The algorithm is initiated from the electronic representation of the Feynman graphs generated with the QGRAF package, [38]. These graphs are adapted by automatically labelling with colour, spinor, Lorentz and flavour indices in a FoRM module prior to applying the projection matrix to isolate the individual scalar amplitudes within several other Form modules. The bulk of the evaluation centres on the integration of the large set of Feynman integrals contributing to a graph after scalar products of internal and external momenta are written in terms of the propagators, respecting the relations (2.6) and (2.7). In arranging the scalar products in this way some integrals are produced with irreducible numerators. By this we mean that there was either no propagator of that form in the original topology or a propagator has a negative power. To proceed requires using integration by parts enshrined in the Laporta algorithm, 39]. This is a technique which systematically constructs algebraic relations between scalar integrals with or without irreducible propagators. The resulting tower of relations, while over-redundant, can then be solved in terms of a minimal number of basic scalar integrals. Termed masters their $\epsilon$-expansion can only be deduced by non-integration by parts methods. All our calculations are carried out using dimensional regularization in $d=4-2 \epsilon$ dimensions. However, for the asymmetric momentum configuration we are considering it transpires that the various master integrals are known to the required order in $\epsilon$ from various sources, [40, 41, 42, 43, 6]. The various polylogarithm functions which appear in the finite part of (2.4) are noted in the next section. For our automatic evaluation we have generated a database of integrals using the REDUZE, [4], implementation of the Laporta algorithm. The output is readily converted into FORM syntax and thence into a FORM module. Once each contributing graph has passed through the integration algorithm they are summed and the Green's function rendered finite. This is carried out automatically using the process provided in [45]. We work with a bare coupling constant and gauge parameter, $\alpha$, in the evaluation of all Feynman diagrams and their renormalized counterparts are introduced by the usual rescaling. This introduces the counterterms without having to perform subtractions which would be difficult to implement in a symbolic manipulation programme. There are various checks on the final expression for the Green's function for each operator. First, we use an arbitrary linear covariant gauge. Therefore, as each operator is gauge invariant its $\overline{\mathrm{MS}}$ renormalization constant will be independent of the gauge fixing parameter $\alpha$. Moreover, it cannot depend on $\omega$ and we note that each operator passed this test as well as agreeing with the known two loop renormalization constants. The other main check was that we recovered the known results, [4, 5, 6, 7, 8, for each Green's function in the $\omega \rightarrow 1$ limit and therefore we are confident that our results are not incorrect.

\section{Basic operators.}

We now turn to the discussion of our results. As a check on our Form code we have reproduced the asymmetric point results of [6] for the scalar current $S$. In particular we found agreement with the conversion function for the anomalous dimension of the mass operator from the $\overline{\mathrm{MS}}$ scheme to the scheme defined in [6]. This scheme was termed the IMOM scheme, [6], to distinguish 
it from the MOM or SMOM schemes. We will use the same nomenclature here. However, as we used a different point of view for the renormalization of $\alpha$ in the $\mathrm{RI}^{\prime}$ scheme aspect of the IMOM scheme the results will only tally in the Landau gauge which is the only gauge of interest for lattice matching anyway. In the attached data file we record the second channel amplitude in addition to that for channel 1 for completeness. One of the main results of [6] was the conversion function. Although this was presented in [6] we record the same quantity here in order to compare the different gauge parameter dependence. We found

$$
\begin{aligned}
C_{\bar{\psi} \psi}(a, \alpha)=1+ & {\left[\Phi_{(1) \omega, \omega} \alpha+3 \Phi_{(1) \omega, \omega}-2 \alpha-8\right] \frac{C_{F} a}{2} } \\
+ & -36 \ln (\omega) \Phi_{(1) \omega, \omega} \alpha \omega C_{F}-12 \ln (\omega) \Phi_{(1) \omega, \omega} \omega C_{A}-60 \ln (\omega) \Phi_{(1) \omega, \omega} \omega C_{F} \\
& +12 \Omega_{(2) \omega, \omega} \omega C_{A}-24 \Omega_{(2) \omega, \omega} \omega C_{F}-24 \Omega_{(2) 1, \omega} \omega C_{A}+48 \Omega_{(2) 1, \omega} \omega C_{F} \\
& +6 \Phi_{(1) \omega, \omega}^{2} \alpha^{2} \omega C_{F}+36 \Phi_{(1) \omega, \omega}^{2} \alpha \omega C_{F}+12 \Phi_{(1) \omega, \omega}^{2} \omega C_{A}-24 \Phi_{(1) \omega, \omega}^{2} C_{A} \\
& +30 \Phi_{(1) \omega, \omega}^{2} \omega C_{F}+48 \Phi_{(1) \omega, \omega}^{2} C_{F}+9 \Phi_{(1) \omega, \omega} \alpha^{2} \omega C_{A}-24 \Phi_{(1) \omega, \omega} \alpha^{2} \omega C_{F} \\
& +42 \Phi_{(1) \omega, \omega} \alpha \omega C_{A}-48 \Phi_{(1) \omega, \omega} \alpha \omega C_{F}+385 \Phi_{(1) \omega, \omega} \omega C_{A}-168 \Phi_{(1) \omega, \omega} \omega C_{F} \\
& -80 \Phi_{(1) \omega, \omega} \omega T_{F} N_{f}-24 \Phi_{(2) \omega, \omega} \alpha \omega C_{F}-24 \Phi_{(2) \omega, \omega} \omega C_{A}-24 \Phi_{(2) \omega, \omega} \omega C_{F} \\
& +24 \Phi_{(2) 1, \omega} \omega^{2} C_{A}-18 \alpha^{2} \omega C_{A}+24 \alpha^{2} \omega C_{F}-84 \alpha \omega C_{A}+96 \alpha \omega C_{F} \\
& \left.+288 \omega \zeta_{3} C_{A}-1285 \omega C_{A}+57 \omega C_{F}+332 \omega T_{F} N_{f}\right] \frac{C_{F} a^{2}}{24 \omega}+O\left(a^{3}\right)
\end{aligned}
$$

where $a$ and $\alpha$ are $\overline{\mathrm{MS}}$ variables and $a=g^{2} /\left(16 \pi^{2}\right)$ in terms of the gauge coupling constant g. Also $C_{F}, C_{A}$ and $T_{F}$ are the usual group theoretic quantities for a Lie group and $\zeta_{z}$ is the Riemann zeta function. Our shorthand notation for various functions is

$$
\begin{aligned}
& \Phi_{(n) 1, \omega}=\Phi_{(n)}(1, \omega) \quad, \quad \Phi_{(n) \omega, \omega}=\Phi_{(n)}\left(\frac{1}{\omega}, \frac{1}{\omega}\right) \\
& \Omega_{(n) 1, \omega}=\Omega_{(n)}(1, \omega) \quad, \quad \Omega_{(n) \omega, \omega}=\Omega_{(n)}\left(\frac{1}{\omega}, \frac{1}{\omega}\right) .
\end{aligned}
$$

These arise from the various underlying master integrals and were evaluated explicitly in terms of polylogarithm functions in 40, 41, 42, 43]. As we will be representing the results for the other operators of interest in graphical form within the article, for completeness we have plotted the channel 1 scalar operator amplitude in Figure 1. There we show the one and two loop corrections as a function of $\omega$ for values of $N_{f}$ with $3 \leq N_{f} \leq 6$ at a specific value of $\alpha_{s}$. This is for illustration as the full results are available for the interested reader in the data file. In Figure 1 and subsequent figures we note that we use the notation $L_{m n l}$ in the legend to denote the amplitude of the operator $L$. The subscript $m n l$ labels the Lorentz channel number, the number of quark flavours and the loop order respectively.

The renormalization of the vector operator requires care due to its underlying significance as a conserved physical current. In other words due to charge conservation in the quantum theory $\partial_{\mu}\left(\bar{\psi} \gamma^{\mu} \psi\right)=0$. So to extract the correct renormalization constant for $V$ one has to express this condition, which equates effectively to the Slavnov-Taylor identity, in the context of the Green's function with the operator inserted at non-zero momentum. A decomposition into the full Lorentz tensor basis is necessary for this. Once the combination of amplitudes is constructed which corresponds to the divergence free current condition then the vector current renormalization constant can be set. Specifically, we require the combination

$$
\left.\Sigma_{(1)}^{V}(p, q)\right|_{\overline{\mathrm{MS}}}-\left.\frac{1}{2} \Sigma_{(2)}^{V}(p, q)\right|_{\overline{\mathrm{MS}}}-\left.\frac{1}{2} \Sigma_{(5)}^{V}(p, q)\right|_{\overline{\mathrm{MS}}}
$$

to be finite in the $\overline{\mathrm{MS}}$ scheme. This is in contrast to the renormalization of the other operators we consider here. In those cases we ensure that the channel in the Lorentz decomposition of the 

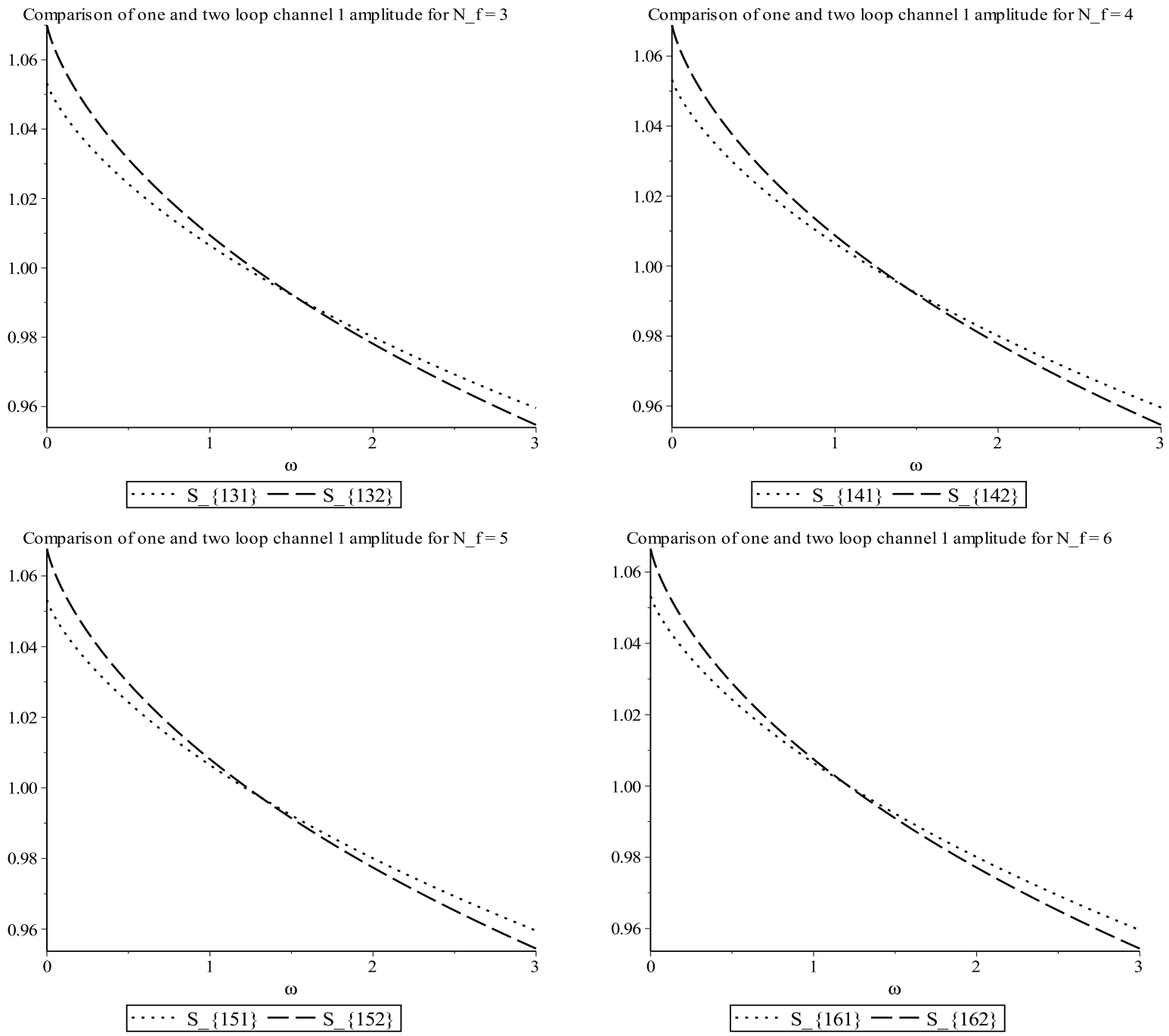

Figure 1: Channel 1 amplitude for scalar operator for different $N_{f}$ values and $\alpha_{s}=0.125$. 
Green's function containing the tree is finite in the $\overline{\mathrm{MS}}$ scheme. However, since the vector current is a physical operator then its renormalization is trivial in all renormalization schemes. In other words $Z^{V}=1$ and consequently $\gamma^{V}(a)=0$ to all orders. For our interpolating subtraction point computation we have checked that this is indeed the case to two loops which plays an important check on the calculation. However, we have summarized the renormalization process for this specific operator to ensure that if others choose to renormalize in a scheme other than $\overline{\mathrm{MS}}$ then the Slavnov-Taylor identity is respected. Another check on the results is that the general relations, [7],

$$
\left.\Sigma_{(2)}^{V}(p, q)\right|_{\omega=1}=\left.\Sigma_{(5)}^{V}(p, q)\right|_{\omega=1} \quad,\left.\quad \Sigma_{(3)}^{V}(p, q)\right|_{\omega=1}=\left.\Sigma_{(4)}^{V}(p, q)\right|_{\omega=1}
$$

have to be satisfied in the $\overline{\mathrm{MS}}$ scheme. These relations follow from the fact that the Green's function is symmetric under the interchange of $p$ and $q$ in the external legs. Their forms were not assumed at the outset but emerged naturally within the computation and acts as a useful check. Given that our amplitudes fulfil the general criterion we have checked that in the $\omega \rightarrow 1$ limit the fully symmetric point results correctly emerge. With these considerations we have plotted the channel 1 amplitudes in Figure 2 for $\alpha_{s}=0.125$. It is evident that for the value of $\alpha_{s}$ the variation from one to two loops across the ranges of $\omega$ and $N_{f}$ is not large. In fact for these ranges it is no more than $0.5 \%$. This is not unexpected since for this particular value of the coupling constant perturbation theory is expected to be a solid approximation.

Finally, we complete this section by considering the tensor current. For its renormalization we have checked that the correct $\omega$-independent two loop $\overline{\mathrm{MS}}$ renormalization constant, [46], first emerges at the $\omega$-dependent subtraction point. The actual divergence in $\epsilon$ being present only in channel 1 as expected from renormalizability. The emergence of the known $\overline{\mathrm{MS}}$ renormalization constant is a useful check on the Form setup. Concerning the other channels a similar left-right symmetry in the original Green's function to the vector case is present corresponding to, [7],

$$
\left.\Sigma_{(3)}^{T}(p, q)\right|_{\omega=1}=\left.\Sigma_{(6)}^{T}(p, q)\right|_{\omega=1} \quad,\left.\quad \Sigma_{(4)}^{T}(p, q)\right|_{\omega=1}=\left.\Sigma_{(5)}^{T}(p, q)\right|_{\omega=1}
$$

in general. We have checked that these amplitudes satisfy the relations at two loops in the $\overline{\mathrm{MS}}$ scheme. An illustration of the behaviour of the channel 1 amplitude is given in Figure 3 . While the form of the plots appear different from the previous two cases it should be noted that the vertical scale is more finely grained in the tensor case. So the behaviour close to $\omega=0$ is amplified.

\section{Wilson operator.}

We devote this section to the results for the second moment of the Wilson operator, $W_{2}$. This is because the renormalization of the two basic operators with the same quantum numbers is not multiplicative unlike the operators of the previous section. Instead $W_{2}$ and $\partial W_{2}$ mix under renormalization and lead to a mixing matrix of anomalous dimensions which we will denote by

$\gamma_{i j}^{W_{2}}(a)$, [8]. It is derived from the set of associated renormalization constants $Z_{i j}^{W_{2}}$ which are defined by

$$
\mathcal{O}_{\mathrm{O} i}=Z_{i j}^{W_{2}} \mathcal{O}_{j}
$$

where the subscript ${ }_{\mathrm{o}}$ indicates a bare quantity. For the two independent operators in the basis we have chosen it transpires that $Z_{i j}^{W_{2}}$ is upper triangular. Specifically, [8,

$$
Z_{i j}^{W_{2}}=\left(\begin{array}{cc}
Z_{11}^{W_{2}} & Z_{12}^{W_{2}} \\
0 & Z_{22}^{W_{2}}
\end{array}\right)
$$



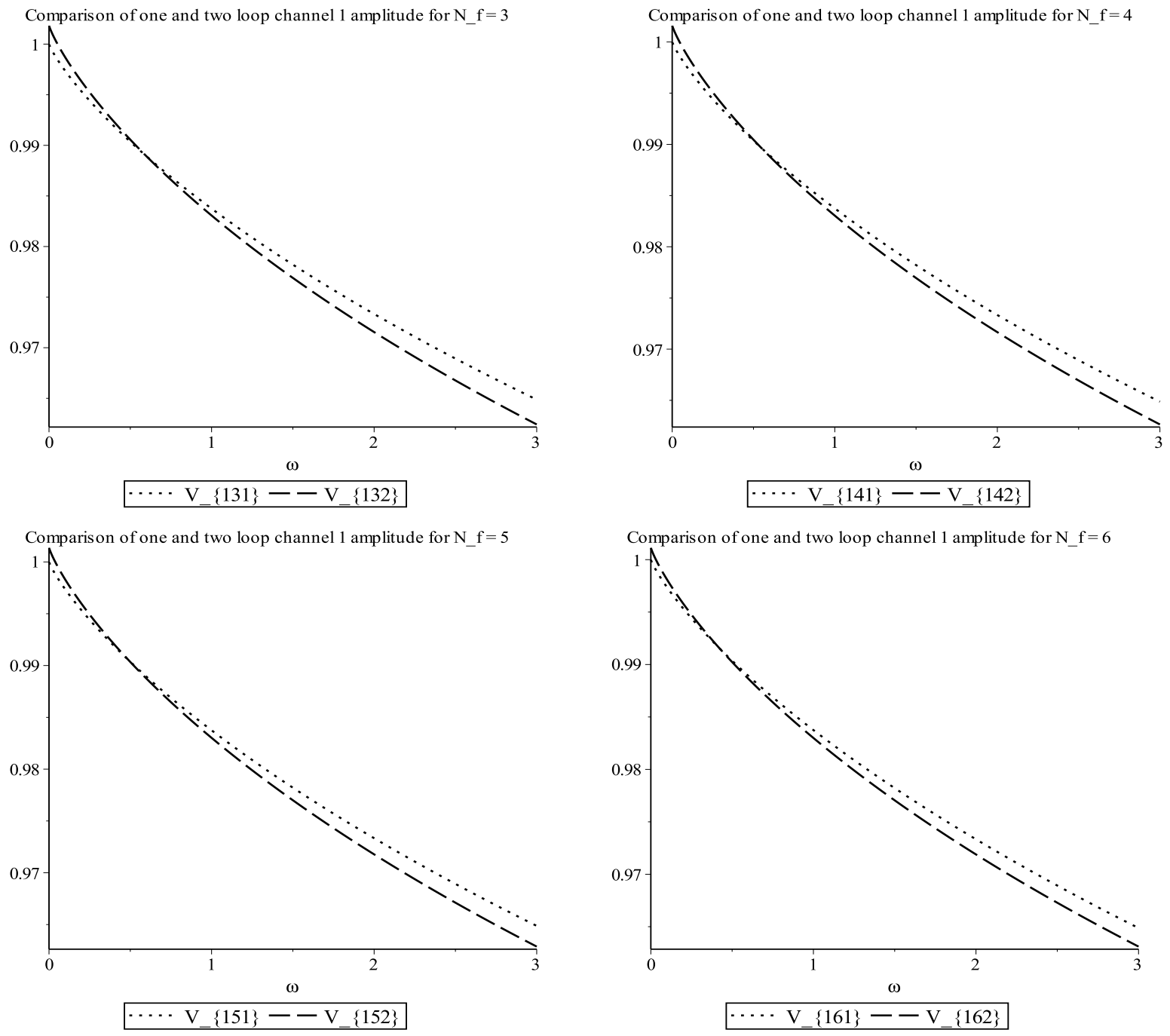

Figure 2: Channel 1 amplitude for vector current for different $N_{f}$ values and $\alpha_{s}=0.125$. 

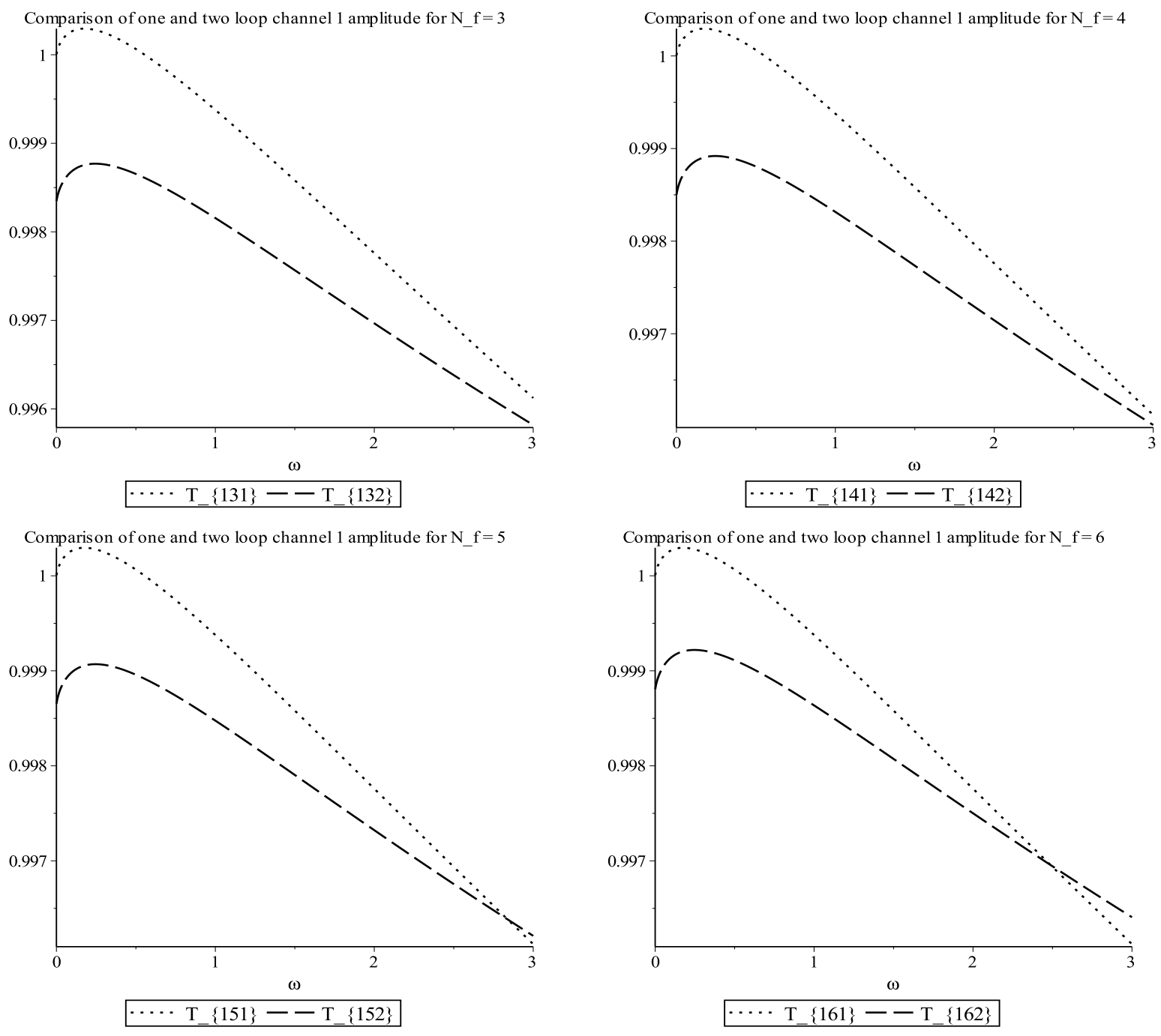

Figure 3: Channel 1 amplitude for tensor operator for different $N_{f}$ values and $\alpha_{s}=0.125$. 
which not only simplifies the renormalization process but allows us to make contact with the usual way the renormalization of the operator $W_{2}$ is derived. For instance, $W_{2}$ is ordinarily regarded as being a multiplicatively renormalizable operator and $\gamma_{11}^{W_{2}}(a)$ is known to three loops in the $\overline{\mathrm{MS}}$ scheme, 1, 2, 3]. However, this multiplicative renormalization is due to the fact that when one renormalizes the operator it is invariably in the situation where it is inserted in a quark 2-point function at zero momentum. This is not the momentum configuration we use here. Therefore, in the zero momentum operator insertion setup the mixing with the total derivative operator is not accessible. This is because momentum conservation would render the Feynman rule for $\partial W_{2}$ to be zero. Another way of putting this is that the full content of the renormalization of the $W_{2}$ sector can only be fully appreciated at a non-exceptional point. An alternative basis to the one chosen here, such as that with the flavour non-singlet operator $\mathcal{S}\left(D^{\mu} \bar{\psi}\right) \gamma^{\nu} \psi$ instead, would lead to a different form of the mixing matrix. So proceeding with our basis choice, [8] and following the projection method given earlier we have constructed all the amplitudes for both operators $W_{2}$ and $\partial W_{2}$ to two loops in the $\overline{\mathrm{MS}}$ scheme. As the first stage we have verified that the correct two loop $\overline{\mathrm{MS}}$ renormalization constants emerge in both cases. For the former operator the tally is with [1, 2]. For $\partial W_{2}$ we have verified that, 8],

$$
\left.\gamma_{22}^{W_{2}}(a)\right|_{\overline{\mathrm{MS}}}=O\left(a^{3}\right)
$$

which is the same as $\gamma^{V}(a)$. This is not unconnected as $\partial W_{2}$ is the total derivative of the nonsinglet vector current. So it is reassuring that this emerges consistently. Partly related to this is that the amplitudes for this operator obey relations similar to (3.4). In particular we have checked to two loops that, [8,

$$
\begin{aligned}
& \left.\Sigma_{(1)}^{\partial W_{2}}(p, q)\right|_{\omega=1}=\left.\Sigma_{(2)}^{\partial W_{2}}(p, q)\right|_{\omega=1} \quad,\left.\quad \Sigma_{(3)}^{\partial W_{2}}(p, q)\right|_{\omega=1}=\left.\Sigma_{(8)}^{\partial W_{2}}(p, q)\right|_{\omega=1} \\
& \left.\Sigma_{(4)}^{\partial W_{2}}(p, q)\right|_{\omega=1}=\left.\Sigma_{(7)}^{\partial W_{2}}(p, q)\right|_{\omega=1} \quad,\left.\quad \Sigma_{(5)}^{\partial W_{2}}(p, q)\right|_{\omega=1}=\left.\Sigma_{(6)}^{\partial W_{2}}(p, q)\right|_{\omega=1} \\
& \left.\Sigma_{(9)}^{\partial W_{2}}(p, q)\right|_{\omega=1}=\left.\Sigma_{(10)}^{\partial W_{2}}(p, q)\right|_{\omega=1}
\end{aligned}
$$

which are established from the left-right symmetry of the underlying Green's function for arbitrary $\omega$. For the operator $W_{2}$ itself there are no similar relations. This is because the covariant derivative in the operator definition only acts on the quark and not the anti-quark field.

While we have presented results for other operators graphically we will do so for $W_{2}$ too. However, as a guide to the form of what lies behind such a representation we provide the channel 2 amplitude in analytic form in the $\overline{\mathrm{MS}}$ scheme. We have

$$
\begin{aligned}
\left.\Sigma_{(2)}^{W_{2}}(p, q)\right|_{\omega} ^{\alpha=0}=- & 1 \\
+ & {\left[\frac{79}{18}+\frac{5}{3} \frac{1}{[\omega-4]}-\frac{17}{6} \ln (\omega)-10 \ln (\omega) \frac{1}{[\omega-4]^{2}}-\frac{28}{3} \ln (\omega) \frac{1}{[\omega-4]}\right.} \\
& \left.+\Phi_{(1) \omega, \omega}-\frac{9}{8} \Phi_{(1) \omega, \omega} \frac{1}{\omega}+\frac{5}{2} \Phi_{(1) \omega, \omega} \frac{1}{[\omega-4]^{2}}+\frac{17}{8} \Phi_{(1) \omega, \omega} \frac{1}{[\omega-4]}\right] C_{F} a \\
+ & {\left[\left[-\frac{2261}{162}-6 \frac{1}{[\omega-4]}+\frac{92}{9} \ln (\omega)+36 \ln (\omega) \frac{1}{[\omega-4]^{2}}+\frac{310}{9} \ln (\omega) \frac{1}{[\omega-4]}\right.\right.} \\
& \quad-\frac{31}{9} \Phi_{(1) \omega, \omega}+\frac{139}{36} \Phi_{(1) \omega, \omega} \frac{1}{\omega}-9 \Phi_{(1) \omega, \omega} \frac{1}{[\omega-4]^{2}} \\
& \left.-\frac{283}{36} \Phi_{(1) \omega, \omega} \frac{1}{[\omega-4]}\right] C_{F} T_{F} N_{f} \\
+ & {\left[\frac{24337}{648}+\frac{241}{18} \frac{1}{[\omega-4]}+18 \zeta_{3} \frac{1}{[\omega-4]^{2}}+21 \zeta_{3} \frac{1}{[\omega-4]}+\zeta_{3}-\frac{2209}{72} \ln (\omega)\right.}
\end{aligned}
$$




$$
\begin{aligned}
& -\frac{241}{3} \ln (\omega) \frac{1}{[\omega-4]^{2}}-\frac{1141}{12} \ln (\omega) \frac{1}{[\omega-4]}-\frac{29}{8} \ln ^{2}(\omega) \\
& -\frac{143}{2} \ln ^{2}(\omega) \frac{1}{[\omega-4]^{2}}-\frac{61}{2} \ln ^{2}(\omega) \frac{1}{[\omega-4]}+\frac{1}{4} \ln (\omega) \Phi_{(1) \omega, \omega} \\
& -\frac{15}{32} \ln (\omega) \Phi_{(1) \omega, \omega} \frac{1}{\omega}+\frac{143}{8} \ln (\omega) \Phi_{(1) \omega, \omega} \frac{1}{[\omega-4]^{2}} \\
& +\frac{271}{32} \ln (\omega) \Phi_{(1) \omega, \omega} \frac{1}{[\omega-4]}-\frac{31}{12} \Omega_{(2) \omega, \omega}-\frac{25}{32} \Omega_{(2) \omega, \omega} \frac{1}{\omega} \\
& -\frac{41}{8} \Omega_{(2) \omega, \omega} \frac{1}{[\omega-4]^{2}}-\frac{737}{96} \Omega_{(2) \omega, \omega} \frac{1}{[\omega-4]}-\frac{17}{3} \Omega_{(2) 1, \omega} \\
& -59 \Omega_{(2) 1, \omega} \frac{1}{[\omega-4]^{2}}-\frac{205}{6} \Omega_{(2) 1, \omega} \frac{1}{[\omega-4]}+\frac{551}{36} \Phi_{(1) \omega, \omega} \\
& -\frac{1039}{72} \Phi_{(1) \omega, \omega} \frac{1}{\omega}+\frac{241}{12} \Phi_{(1) \omega, \omega} \frac{1}{[\omega-4]^{2}}+\frac{2683}{72} \Phi_{(1) \omega, \omega} \frac{1}{[\omega-4]} \\
& +\frac{1}{2} \Phi_{(1) \omega, \omega}^{2}-\frac{1}{2} \Phi_{(1) \omega, \omega}^{2} \frac{1}{\omega}-2 \Phi_{(2) \omega, \omega}+\frac{25}{16} \Phi_{(2) \omega, \omega} \frac{1}{\omega^{2}} \\
& +\frac{29}{16} \Phi_{(2) \omega, \omega} \frac{1}{\omega}-\frac{29}{16} \Phi_{(2) \omega, \omega} \frac{1}{[\omega-4]^{2}}-\frac{45}{16} \Phi_{(2) \omega, \omega} \frac{1}{[\omega-4]} \\
& +26 \Phi_{(2) 1, \omega}+224 \Phi_{(2) 1, \omega} \frac{1}{[\omega-4]^{2}}+160 \Phi_{(2) 1, \omega} \frac{1}{[\omega-4]} \\
& \left.+3 \Phi_{(2) 1, \omega} \omega\right] C_{F} C_{A} \\
& +\left[-\frac{10861}{648}-\frac{44}{9} \frac{1}{[\omega-4]}-120 \zeta_{3} \frac{1}{[\omega-4]^{2}}-112 \zeta_{3} \frac{1}{[\omega-4]}-34 \zeta_{3}\right. \\
& +\frac{221}{12} \ln (\omega)+\frac{88}{3} \ln (\omega) \frac{1}{[\omega-4]^{2}}+\frac{1001}{18} \ln (\omega) \frac{1}{[\omega-4]}+\frac{65}{18} \ln (\omega)^{2} \\
& +\frac{454}{3} \ln ^{2}(\omega) \frac{1}{[\omega-4]^{2}}+\frac{490}{9} \ln ^{2}(\omega) \frac{1}{[\omega-4]}+\frac{4}{3} \ln (\omega) \Phi_{(1) \omega, \omega} \\
& -\frac{13}{24} \ln (\omega) \Phi_{(1) \omega, \omega} \frac{1}{\omega}-\frac{227}{6} \ln (\omega) \Phi_{(1) \omega, \omega} \frac{1}{[\omega-4]^{2}} \\
& -\frac{379}{24} \ln (\omega) \Phi_{(1) \omega, \omega} \frac{1}{[\omega-4]}+\frac{8}{3} \Omega_{(2) \omega, \omega}+\frac{3}{4} \Omega_{(2) \omega, \omega} \frac{1}{\omega}+\Omega_{(2) \omega, \omega} \frac{1}{[\omega-4]^{2}} \\
& +\frac{79}{12} \Omega_{(2) \omega, \omega} \frac{1}{[\omega-4]}+\frac{40}{3} \Omega_{(2) 1, \omega}+148 \Omega_{(2) 1, \omega} \frac{1}{[\omega-4]^{2}} \\
& +\frac{244}{3} \Omega_{(2) 1, \omega} \frac{1}{[\omega-4]}-\frac{679}{36} \Phi_{(1) \omega, \omega}+\frac{487}{72} \Phi_{(1) \omega, \omega} \frac{1}{\omega} \\
& -\frac{22}{3} \Phi_{(1) \omega, \omega} \frac{1}{[\omega-4]^{2}}-\frac{4039}{72} \Phi_{(1) \omega, \omega} \frac{1}{[\omega-4]}-\Phi_{(1) \omega, \omega}^{2}+\Phi_{(1) \omega, \omega}^{2} \frac{1}{\omega} \\
& -\frac{3}{2} \Phi_{(2) \omega, \omega} \frac{1}{\omega^{2}}+\Phi_{(2) \omega, \omega} \frac{1}{\omega}-\frac{9}{2} \Phi_{(2) \omega, \omega} \frac{1}{[\omega-4]^{2}}-\Phi_{(2) \omega, \omega} \frac{1}{[\omega-4]} \\
& -52 \Phi_{(2) 1, \omega}-512 \Phi_{(2) 1, \omega} \frac{1}{[\omega-4]^{2}}-336 \Phi_{(2) 1, \omega} \frac{1}{[\omega-4]} \\
& \left.\left.-4 \Phi_{(2) 1, \omega} \omega\right] C_{F}^{2}\right] a^{2}+O\left(a^{3}\right) .
\end{aligned}
$$

The reason for concentrating on channel 2 is that that is the channel which corresponds to the Feynman rule for $W_{2}$. By contrast the channel 1 basis tensor involves the external momentum $p$ which would be associated with the covariant derivative in the operator $\mathcal{S}\left(D^{\mu} \bar{\psi}\right) \gamma^{\nu} \psi$ which is not in our basis. Graphically we have presented the results for channel 2 in Figure 4. Although strictly we have plotted the negative of (4.5) in order to easily compare with the Figures of the 

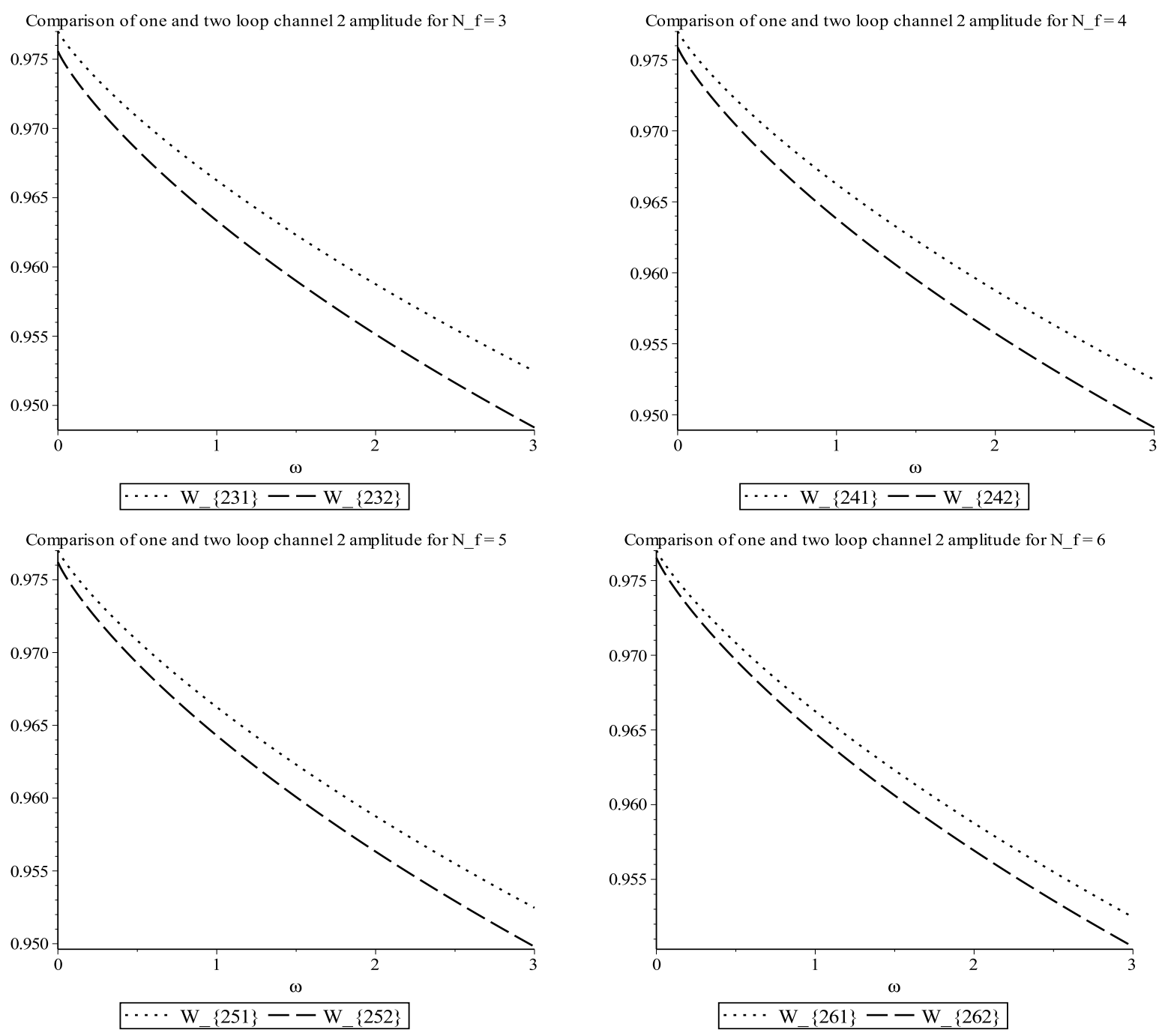

Figure 4: Channel 2 amplitude for $W_{2}$ operator for different $N_{f}$ values and $\alpha_{s}=0.125$.

previous section. In essence they are formally similar to earlier representations. For instance, as $N_{f}$ increases there is less variation between the one and two loop amplitudes as a function of $\omega$ for the value of $\alpha_{s}$ considered. Finally, we have checked that the analogous plots for $\partial W_{2}$ are the same as those for the vector case of the previous section. This is not unexpected given the structural similarity of the two operators. Therefore, we have not produced parallel graphs for $\partial W_{2}$.

\section{Discussion.}

We finish with brief observations. The inclusion of a parameter, $\omega$, which allows one to tune the momentum flow through the operator inserted in the basic Green's function should prove useful for lattice analyses. For instance, it provides a tool with which one can reduce the errors on measurements of structure functions. Although from the various graphs the variation on the highlighted amplitudes over the range of $\omega$ plotted is not the same overall. For instance, for the 
vector and tensor operators the two loop correction is virtually insignificant compared to the scalar case. For the vector this is important in the context of $W_{2}$ itself because the behaviour of the vector current amplitudes are effectively the same as those for $\partial W_{2}$. The mixing between these operators has to be disentangled to obtain a clear signal for the basic operator underlying the nucleon structure function measurements. That the variation between one and two loops is small suggests that an error estimate could be commensurate. The present computations complete the two loop analysis of operator renormalization which was begun in [4, 5, 6, 7, 8, The next stage in any programme of this nature would be to go to the next loop order in order to improve the precision even more. This is a major step as one requires the underlying three loop master integrals.

Acknowledgements. This work was carried out with the support in part from the STFC Consolidated Grant ST/L000431/1 and STFC studentship (JMB) as well as through a John Lennon Memorial Scholarship (JMB). The authors thanks R. Horsley, P.E.L. Rakow and G. Schierholz for useful discussions.

\section{A Bases and projection matrices.}

In this appendix we record the full forms of the basis tensors for the Green's function with the operator insertion as well as the projection matrices. As we are using quark operators we have to be aware that the basis tensors do not only depend on $\eta_{\mu \nu}$ and the two independent external momenta $p$ and $q$ but also products of $\gamma$-matrices. The latter can also carry free Lorentz indices but are necessary in order to carry the spinor indices. In order to achieve this we use the generalized $d$-dimensional $\gamma$-matrices defined by, [47, 48, 49, 50, 51,

$$
\Gamma_{(n)}^{\mu_{1} \ldots \mu_{n}}=\gamma^{\left[\mu_{1}\right.} \ldots \gamma^{\left.\mu_{n}\right]}
$$

Our convention is that the $1 / n$ ! factor is included in the antisymmetrization. As these objects are totally antisymmetric in the Lorentz indices then the tensor basis for various operators have natural partitions which is manifested in results such as

$$
\operatorname{tr}\left(\Gamma_{(m)}^{\mu_{1} \ldots \mu_{m}} \Gamma_{(n)}^{\nu_{1} \ldots \nu_{n}}\right) \propto \delta_{m n} I^{\mu_{1} \ldots \mu_{m} \nu_{1} \ldots \nu_{n}}
$$

where $I^{\mu_{1} \ldots \mu_{m} \nu_{1} \ldots \nu_{n}}$ is the generalized unit matrix, [49, 50, 51. Equipped with these structures the basic tensors are in essence the same as those for the symmetric point but the projection matrices are $\omega$ dependent. In the $\omega \rightarrow 1$ limit we have checked that the matrices of [7, 8] are reproduced. For the scalar, vector and tensor operators the bases are, [7,

$$
\begin{gathered}
\mathcal{P}_{(1)}^{S}(p, q)=\Gamma_{(0)}, \quad \mathcal{P}_{(2)}^{S}(p, q)=\frac{1}{\mu^{2}} \Gamma_{(2)}^{p q} \\
\mathcal{P}_{(1) \mu}^{V}(p, q)=\gamma_{\mu}, \quad \mathcal{P}_{(2) \mu}^{V}(p, q)=\frac{p^{\mu} p}{\mu^{2}}, \quad \mathcal{P}_{(3) \mu}^{V}(p, q)=\frac{p_{\mu} \phi}{\mu^{2}}, \\
\mathcal{P}_{(4) \mu}^{V}(p, q)=\frac{q_{\mu} \not p}{\mu^{2}}, \quad \mathcal{P}_{(5) \mu}^{V}(p, q)=\frac{q_{\mu} \phi}{\mu^{2}}, \quad \mathcal{P}_{(6) \mu}^{V}(p, q)=\frac{1}{\mu^{2}} \Gamma_{(3) \mu p q}
\end{gathered}
$$

and

$$
\mathcal{P}_{(1) \mu \nu}^{T}(p, q)=\Gamma_{(2) \mu \nu} \quad, \quad \mathcal{P}_{(2) \mu \nu}^{T}(p, q)=\frac{1}{\mu^{2}}\left[p_{\mu} q_{\nu}-p_{\nu} q_{\mu}\right] \Gamma_{(0)},
$$




$$
\begin{aligned}
& \mathcal{P}_{(3) \mu \nu}^{T}(p, q)=\frac{1}{\mu^{2}}\left[\Gamma_{(2) \mu p} p_{\nu}-\Gamma_{(2) \nu p} p_{\mu}\right], \quad \mathcal{P}_{(4) \mu \nu}^{T}(p, q)=\frac{1}{\mu^{2}}\left[\Gamma_{(2) \mu p} q_{\nu}-\Gamma_{(2) \nu p} q_{\mu}\right], \\
& \mathcal{P}_{(5) \mu \nu}^{T}(p, q)=\frac{1}{\mu^{2}}\left[\Gamma_{(2) \mu q} p_{\nu}-\Gamma_{(2) \nu q} p_{\mu}\right], \quad \mathcal{P}_{(6) \mu \nu}^{T}(p, q)=\frac{1}{\mu^{2}}\left[\Gamma_{(2) \mu q} q_{\nu}-\Gamma_{(2) \nu q} q_{\mu}\right], \\
& \mathcal{P}_{(7) \mu \nu}^{T}(p, q)=\frac{1}{\mu^{4}}\left[\Gamma_{(2) p q} p_{\mu} q_{\nu}-\Gamma_{(2) p q} p_{\nu} q_{\mu}\right] \quad, \quad \mathcal{P}_{(8) \mu \nu}^{T}(p, q)=\frac{1}{\mu^{2}} \Gamma_{(4) \mu \nu p q}
\end{aligned}
$$

respectively where the contraction of a $\Gamma$-matrix with an external momentum is represented by the momentum itself replacing the contracting index. The situation for the Wilson operator is slightly different. As in [8] we choose a set of tensors which have the same symmetry properties as the operators themselves. However, in order to ensure tracelessness the associated contractions in certain tensors involves $p q$ and hence $\omega$. So unlike $S, V$ and $T$ certain tensors for the $W_{2}$ and $\partial W_{2}$ decomposition are $\omega$ dependent. In particular

$$
\begin{aligned}
\mathcal{P}_{(1) \mu \nu}^{W_{2}}(p, q) & =\gamma_{\mu} p_{\nu}+\gamma_{\nu} p_{\mu}-\frac{2}{d} \not p \eta_{\mu \nu}, \mathcal{P}_{(2) \mu \nu}^{W_{2}}(p, q)=\gamma_{\mu} q_{\nu}+\gamma_{\nu} q_{\mu}-\frac{2}{d} \not \eta_{\mu \nu} \\
\mathcal{P}_{(3) \mu \nu}^{W_{2}}(p, q) & \left.\left.=\not \frac{1}{\mu^{2}} p_{\mu} p_{\nu}+\frac{1}{d} \eta_{\mu \nu}\right], \quad \mathcal{P}_{(4) \mu \nu}^{W_{2}}(p, q)=\not \frac{1}{\mu^{2}} p_{\mu} q_{\nu}+\frac{1}{\mu^{2}} q_{\mu} p_{\nu}-\frac{(2-\omega)}{d} \eta_{\mu \nu}\right] \\
\mathcal{P}_{(5) \mu \nu}^{W_{2}}(p, q) & =\not p\left[\frac{1}{\mu^{2}} q_{\mu} q_{\nu}+\frac{1}{d} \eta_{\mu \nu}\right], \mathcal{P}_{(6) \mu \nu}^{W_{2}}(p, q)=\not\left[\frac{1}{\mu^{2}} p_{\mu} p_{\nu}+\frac{1}{d} \eta_{\mu \nu}\right] \\
\mathcal{P}_{(7) \mu \nu}^{W_{2}}(p, q) & =\not q\left[\frac{1}{\mu^{2}} p_{\mu} q_{\nu}+\frac{1}{\mu^{2}} q_{\mu} p_{\nu}-\frac{(2-\omega)}{d} \eta_{\mu \nu}\right], \mathcal{P}_{(8) \mu \nu}^{W_{2}}(p, q)=\not q\left[\frac{1}{\mu^{2}} q_{\mu} q_{\nu}+\frac{1}{d} \eta_{\mu \nu}\right] \\
\mathcal{P}_{(9) \mu \nu}^{W_{2}}(p, q) & =\frac{1}{\mu^{2}}\left[\Gamma_{(3) \mu p q} p_{\nu}+\Gamma_{(3) \nu p q} p_{\mu}\right] \\
\mathcal{P}_{(10) \mu \nu}^{W_{2}}(p, q) & =\frac{1}{\mu^{2}}\left[\Gamma_{(3) \mu p q} q_{\nu}+\Gamma_{(3) \nu p q} q_{\mu}\right] .
\end{aligned}
$$

We note that in the renormalization of the operator $W_{2}$ the basis element corresponding to the tree term of (2.4) is channel 2 and not 1 . This is because the covariant derivative acts on the quark field and not the anti-quark. For $\partial W_{2}$ both channels 1 and 2 are relevant to the operator renormalization.

Equipped with these the various projection matrices are

$$
\mathcal{M}^{S}=\frac{1}{4 \omega[\omega-4]}\left(\begin{array}{cc}
\omega[\omega-4] & 0 \\
0 & 4
\end{array}\right)
$$

for the scalar case. For the remaining operators since the projection matrices are symmetric by construction we list the upper triangular elements only. For the vector operator if we factor off a common factor, which is not the determinant of $\mathcal{N}^{V}$, via

$$
\mathcal{M}^{V}=\frac{1}{4(d-2) \omega^{2}[\omega-4]^{2}} \tilde{\mathcal{M}}^{V}
$$

then the elements are

$$
\begin{aligned}
& \tilde{\mathcal{M}}_{11}^{V}=[\omega-4]^{2} \omega^{2}, \quad \tilde{\mathcal{M}}_{12}^{V}=-4[\omega-4] \omega, \tilde{\mathcal{M}}_{13}^{V}=2[\omega-2][\omega-4] \omega \\
& \tilde{\mathcal{M}}_{14}^{V}=2[\omega-2][\omega-4] \omega, \quad \tilde{\mathcal{M}}_{15}^{V}=-4[\omega-4] \omega, \quad \tilde{\mathcal{M}}_{16}^{V}=0, \quad \tilde{\mathcal{M}}_{22}^{V}=16[d-1] \\
& \tilde{\mathcal{M}}_{23}^{V}=-8[d-1][\omega-2], \quad \tilde{\mathcal{M}}_{24}^{V}=-8[d-1][\omega-2] \\
& \tilde{\mathcal{M}}_{25}^{V}=-4\left[2\left[\omega^{2}-4 \omega+2\right]-[\omega-2]^{2} d\right], \quad \tilde{\mathcal{M}}_{26}^{V}=0, \quad \tilde{\mathcal{M}}_{33}^{V}=4\left[\omega^{2}-4 \omega-4+4 d\right] \\
& \tilde{\mathcal{M}}_{34}^{V}=4[d-1][\omega-2]^{2}, \quad \tilde{\mathcal{M}}_{35}^{V}=-8[d-1][\omega-2], \quad \tilde{\mathcal{M}}_{36}^{V}=0 \\
& \tilde{\mathcal{M}}_{44}^{V}=4\left[\omega^{2}-4 \omega-4+4 d\right], \quad \tilde{\mathcal{M}}_{45}^{V}=-8[d-1][\omega-2], \quad \tilde{\mathcal{M}}_{46}^{V}=0 \\
& \tilde{\mathcal{M}}_{55}^{V}=16[d-1], \quad \tilde{\mathcal{M}}_{56}^{V}=0, \quad \tilde{\mathcal{M}}_{66}^{V}=4[\omega-4] \omega .
\end{aligned}
$$


For such a simple spin-1 operator the $\omega$ dependence is quite involved. In the tensor case, with

$$
\mathcal{M}^{T}=\frac{1}{4(d-2)(d-3) \omega^{2}[\omega-4]^{2}} \tilde{\mathcal{M}}^{T}
$$

then

$$
\begin{aligned}
& \tilde{\mathcal{M}}_{11}^{T}=-[\omega-4]^{2} \omega^{2}, \quad \tilde{\mathcal{M}}_{12}^{T}=0, \quad \tilde{\mathcal{M}}_{13}^{T}=4[\omega-4] \omega, \tilde{\mathcal{M}}_{14}^{T}=-2[\omega-2][\omega-4] \omega \\
& \tilde{\mathcal{M}}_{15}^{T}=-2[\omega-2][\omega-4] \omega, \quad \tilde{\mathcal{M}}_{16}^{T}=4[\omega-4] \omega, \tilde{\mathcal{M}}_{17}^{T}=4[\omega-4] \omega, \quad \tilde{\mathcal{M}}_{18}^{T}=0 \\
& \tilde{\mathcal{M}}_{22}^{T}=-2[d-2][d-3][\omega-4] \omega, \tilde{\mathcal{M}}_{23}^{T}=0, \tilde{\mathcal{M}}_{24}^{T}=0, \tilde{\mathcal{M}}_{25}^{T}=0, \quad \tilde{\mathcal{M}}_{26}^{T}=0 \\
& \tilde{\mathcal{M}}_{27}^{T}=0, \quad \tilde{\mathcal{M}}_{28}^{T}=0, \quad \tilde{\mathcal{M}}_{33}^{T}=-8[d-1], \quad \tilde{\mathcal{M}}_{34}^{T}=4[d-1][\omega-2] \\
& \tilde{\mathcal{M}}_{35}^{T}=4[d-1][\omega-2], \quad \tilde{\mathcal{M}}_{36}^{T}=-2\left[d \omega^{2}-4 d \omega+4 d-3 \omega^{2}+12 \omega-4\right] \\
& \tilde{\mathcal{M}}_{37}^{T}=-8[d-1], \quad \tilde{\mathcal{M}}_{38}^{T}=0, \quad \tilde{\mathcal{M}}_{44}^{T}=-4\left[2 d+\omega^{2}-4 \omega-2\right] \\
& \tilde{\mathcal{M}}_{45}^{T}=-2[d-1][\omega-2]^{2}, \quad \tilde{\mathcal{M}}_{46}^{T}=4[d-1][\omega-2], \quad \tilde{\mathcal{M}}_{47}^{T}=4[d-1][\omega-2] \\
& \tilde{\mathcal{M}}_{48}^{T}=0, \quad \tilde{\mathcal{M}}_{55}^{T}=-4\left[2 d+\omega^{2}-4 \omega-2\right], \quad \tilde{\mathcal{M}}_{56}^{T}=4[d-1][\omega-2] \\
& \tilde{\mathcal{M}}_{57}^{T}=4[d-1][\omega-2], \quad \tilde{\mathcal{M}}_{58}^{T}=0, \quad \tilde{\mathcal{M}}_{66}^{T}=-8[d-1], \quad \tilde{\mathcal{M}}_{67}^{T}=-8[d-1] \\
& \tilde{\mathcal{M}}_{68}^{T}=0, \quad \tilde{\mathcal{M}}_{77}^{T}=-8[d-1][d-2], \quad \tilde{\mathcal{M}}_{78}^{T}=0, \quad \tilde{\mathcal{M}}_{88}^{T}=-4[\omega-4] \omega .
\end{aligned}
$$

Finally, for the twist-2 operator we set

$$
\mathcal{M}^{W_{2}}=\frac{1}{4(d-2) \omega^{3}[\omega-4]^{3}} \tilde{\mathcal{M}}^{W_{2}}
$$

to produce the elements

$$
\begin{aligned}
& \tilde{\mathcal{M}}_{11}^{W_{2}}=2[\omega-4]^{2} \omega^{2}, \quad \tilde{\mathcal{M}}_{12}^{W_{2}}=-[\omega-2][\omega-4]^{2} \omega^{2}, \quad \tilde{\mathcal{M}}_{13}^{W_{2}}=-16[\omega-4] \omega \\
& \tilde{\mathcal{M}}_{14}^{W_{2}}=8[\omega-2][\omega-4] \omega, \quad \tilde{\mathcal{M}}_{15}^{W_{2}}=-4[\omega-2]^{2}[\omega-4] \omega, \quad \tilde{\mathcal{M}}_{16}^{W_{2}}=8[\omega-2][\omega-4] \omega \\
& \tilde{\mathcal{M}}_{17}^{W_{2}}=-2\left[\omega^{2}-4 \omega+8\right][\omega-4] \omega, \quad \tilde{\mathcal{M}}_{18}^{W_{2}}=8[\omega-2][\omega-4] \omega, \quad \tilde{\mathcal{M}}_{19}^{W_{2}}=0 \\
& \tilde{\mathcal{M}}_{110}^{W_{2}}=0, \quad \tilde{\mathcal{M}}_{22}^{W_{2}}=2[\omega-4]^{2} \omega^{2}, \quad \tilde{\mathcal{M}}_{23}^{W_{2}}=8[\omega-2][\omega-4] \omega \\
& \tilde{\mathcal{M}}_{24}^{W_{2}}=-2\left[\omega^{2}-4 \omega+8\right][\omega-4] \omega, \quad \tilde{\mathcal{M}}_{25}^{W_{2}}=8[\omega-2][\omega-4] \omega \\
& \tilde{\mathcal{M}}_{26}^{W_{2}}=-4[\omega-2]^{2}[\omega-4] \omega, \quad \tilde{\mathcal{M}}_{27}^{W_{2}}=8[\omega-2][\omega-4] \omega, \quad \tilde{\mathcal{M}}_{28}^{W_{2}}=-16[\omega-4] \omega \\
& \tilde{\mathcal{M}}_{29}^{W_{2}}=0, \tilde{\mathcal{M}}_{210}^{W_{2}}=0, \tilde{\mathcal{M}}_{33}^{W_{2}}=64[d+1], \tilde{\mathcal{M}}_{34}^{W_{2}}=-32[d+1][\omega-2] \\
& \tilde{\mathcal{M}}_{35}^{W_{2}}=16\left[d \omega^{2}-4 d \omega+4 d+4\right], \quad \tilde{\mathcal{M}}_{36}^{W_{2}}=-32[d+1][\omega-2] \\
& \tilde{\mathcal{M}}_{37}^{W_{2}}=16\left[d \omega^{2}-4 d \omega+4 d+4\right], \quad \tilde{\mathcal{M}}_{38}^{W_{2}}=-8\left[d \omega^{2}-4 d \omega+4 d-2 \omega^{2}+8 \omega+4\right][\omega-2] \\
& \tilde{\mathcal{M}}_{39}^{W_{2}}=0, \quad \tilde{\mathcal{M}}_{310}^{W_{2}}=0, \quad \tilde{\mathcal{M}}_{44}^{W_{2}}-8\left[d \omega^{2}-4 d \omega+8 d+3 \omega^{2}-12 \omega+8\right] \\
& \tilde{\mathcal{M}}_{45}^{W_{2}}=-8\left[4 d+\omega^{2}-4 \omega+4\right][\omega-2], \quad \tilde{\mathcal{M}}_{46}^{W_{2}}=16[d+1][\omega-2]^{2} \\
& \tilde{\mathcal{M}}_{47}^{W_{2}}=-4[d+1]\left[\omega^{2}-4 \omega+8\right][\omega-2], \quad \tilde{\mathcal{M}}_{48}^{W_{2}}=16\left[d \omega^{2}-4 d \omega+4 d+4\right] \\
& \tilde{\mathcal{M}}_{49}^{W_{2}}=0, \tilde{\mathcal{M}}_{410}^{W_{2}}=0, \tilde{\mathcal{M}}_{55}^{W_{2}}=32\left[2 d+\omega^{2}-4 \omega+2\right] \\
& \tilde{\mathcal{M}}_{56}^{W_{2}}=-8\left[d \omega^{2}-4 d \omega+4 d+4\right][\omega-2], \quad \tilde{\mathcal{M}}_{57}^{W_{2}}=16[d+1][\omega-2]^{2} \\
& \tilde{\mathcal{M}}_{58}^{W_{2}}=-32[d+1][\omega-2], \quad \tilde{\mathcal{M}}_{59}^{W_{2}}=0, \quad \tilde{\mathcal{M}}_{510}^{W_{2}}=0 \quad, \quad \tilde{\mathcal{M}}_{66}^{W_{2}}=32\left[2 d+\omega^{2}-4 \omega+2\right] \\
& \tilde{\mathcal{M}}_{67}^{W_{2}}=-8\left[4 d+\omega^{2}-4 \omega+4\right][\omega-2], \quad \tilde{\mathcal{M}}_{68}^{W_{2}}=16\left[d \omega^{2}-4 d \omega+4 d+4\right] \quad, \quad \tilde{\mathcal{M}}_{69}^{W_{2}}=0 \\
& \tilde{\mathcal{M}}_{610}^{W_{2}}=0, \quad \tilde{\mathcal{M}}_{77}^{W_{2}}=8\left[d \omega^{2}-4 d \omega+8 d+3 \omega^{2}-12 \omega+8\right], \quad \tilde{\mathcal{M}}_{78}^{W_{2}}=-32[d+1][\omega-2] \\
& \tilde{\mathcal{M}}_{79}^{W_{2}}=0, \tilde{\mathcal{M}}_{710}^{W_{2}}=0, \quad \tilde{\mathcal{M}}_{88}^{W_{2}}=64[d+1], \quad \tilde{\mathcal{M}}_{89}^{W_{2}}=0, \tilde{\mathcal{M}}_{810}^{W_{2}}=0 \\
& \tilde{\mathcal{M}}_{99}^{W_{2}}=8[\omega-4] \omega, \quad \tilde{\mathcal{M}}_{910}^{W_{2}}=-4[\omega-2][\omega-4] \omega, \quad \tilde{\mathcal{M}}_{1010}^{W_{2}}=8[\omega-4] \omega .
\end{aligned}
$$

For each case the $\omega \rightarrow 0$ limit of the projection matrices are singular as expected since this is a point where there are infrared singularities. 


\section{References.}

[1] D.J. Gross \& F.J. Wilczek, Phys. Rev. D9 (1974), 980.

[2] E.G. Floratos, D.A. Ross \& C.T. Sachrajda, Nucl. Phys. B129 (1977), 66; B139 (1978), $545(\mathrm{E})$.

[3] S. Moch, J.A.M. Vermaseren \& A. Vogt, Nucl. Phys. B688 (2004), 101.

[4] C. Sturm, Y. Aoki, N.H. Christ, T. Izubuchi, C.T.C. Sachrajda \& A. Soni, Phys. Rev. D80 (2009), 014501.

[5] L.G. Almeida \& C. Sturm, Phys. Rev. D82 (2010), 054017.

[6] M. Gorbahn \& S. Jäger, Phys. Rev. D82 (2010), 114001.

[7] J.A. Gracey, Eur. Phys. J. C71 (2011), 1567.

[8] J.A. Gracey, JHEP 1103 (2011), 109.

[9] V. Gimenez, L. Giusti, F. Rapuano \& M. Talevi, Nucl. Phys. B531 (1998), 429.

[10] M. Göckeler, R. Horsley, H. Oelrich, H. Perlt, D. Petters, P.E.L. Rakow, A. Schäfer, G. Schierholz \& A. Schiller, Nucl. Phys. B544 (1999), 699.

[11] S. Capitani, M. Göckeler, R. Horsley, H. Perlt, P.E.L. Rakow, G. Schierholz \& A. Schiller, Nucl. Phys. B593 (2001), 183.

[12] L. Giusti, S. Petrarca, B. Taglienti \& N. Tantalo, Phys. Lett. B541 (2002), 350.

[13] M. Göckeler, R. Horsley, D. Pleiter, P.E.L. Rakow, A. Schäfer and G. Schierholz, Nucl. Phys. Proc. Suppl. 119 (2003), 32.

[14] J.B. Zhang, D.B. Leinweber, K.F. Liu \& A.G. Williams, Nucl. Phys. Proc. Suppl. 128 (2004), 240.

[15] D. Bećirević, V. Gimenez, V. Lubicz, G. Martinelli, M. Papinutto \& J. Reyes, JHEP 0408 (2004), 022.

[16] C. Gattringer, M. Göckeler, P. Huber \& C.B. Lang, Nucl. Phys. B694 (2004), 170.

[17] M. Göckeler, R. Horsley, D. Pleiter, P.E.L. Rakow \& G. Schierholz, Phys. Rev. D71 (2005), 114511.

[18] J.B. Zhang, N. Mathur, S.J. Dong, T. Draper, I. Horvath, F.X. Lee, D.B. Leinweber, K.F. Liu \& A.G. Williams, Phys. Rev. D72 (2005), 114509.

[19] F. Di Renzo, A. Mantovi, V. Miccio, C. Torrero \& L. Scorzato, PoS LAT2005 (2006), 237.

[20] M. Gürtler, R. Horsley, P.E.L. Rakow, C.J. Roberts, G. Schierholz \& T. Streuer, PoS (LAT2005) 125 (2006), 124.

[21] A. Skouroupathis \& H. Panagopoulos, Phys. Rev. D79 (2009), 094508.

[22] M. Göckeler, R. Horsley, Y. Nakamura, H. Perlt, D. Pleiter, P.E.L. Rakow, A. Schäfer, G. Schierholz, A. Schiller, H. Stüben \& J.M. Zanotti, Phys. Rev D82 (2010), 114511. 
[23] M. Constantinou, P. Dimopoulos, R. Frezzotti, G. Herdoiza, K. Jansen, V. Lubicz, H. Panagopoulos, G.C. Rossi, S. Simula, F. Stylianou \& A. Vladikas, JHEP 1008 (2010), 068.

[24] Y. Aoki, PoS (LATTICE2009) (2009), 012.

[25] R. Arthur \& P.A. Boyle, Phys. Rev. D83 (2011), 114511.

[26] C. Alexandrou, M. Constantinou, T. Korzec, H. Panagopoulos \& F. Stylianou, Phys. Rev. D83 (2011), 014503.

[27] R. Arthur \& P.A. Boyle, N. Garron, C. Kelly \& A.T. Lytle, Phys. Rev. D85 (2012), 014501.

[28] Z. Liu, Y. Chen, S.-J. Dong, M. Glatzmaier, M. Gong, A. Li, K.-F. Liu, Y.-B. Yang \& J.-B. Zhang, Phys. Rev. D90 (2014), 034505.

[29] J. Flynn, R. Arthur, P. Boyle, A. Jüttner, C. Sachrajda \& T. Rae, PoS (LATTICE2014) (2014), 168.

[30] V.M. Braun, S. Collins, M. Göckeler, P. Pérez-Rubio, A. Schäfer, R.W. Schiel \& A. Sternbeck, Phys. Rev. D92 (2015), 014504.

[31] D. Becirevic, V. Lubicz, F. Mescia \& C. Tarantino, JHEP 0305 (2003), 007.

[32] G. Martinelli, C. Pittori, C.T. Sachrajda, M. Testa \& A. Vladikas, Nucl. Phys. B445 (1995), 81.

[33] E. Franco \& V. Lubicz, Nucl. Phys. B531 (1998), 641

[34] W. Celmaster \& R.J. Gonsalves, Phys. Rev. Lett. 42 (1979), 1435.

[35] W. Celmaster \& R.J. Gonsalves, Phys. Rev. D20 (1979), 1420.

[36] J.A.M. Vermaseren, math-ph/0010025.

[37] M. Tentyukov \& J.A.M. Vermaseren, Comput. Phys. Commun. 181 (2010), 1419.

[38] P. Nogueira, J. Comput. Phys. 105 (1993), 279.

[39] S. Laporta, Int. J. Mod. Phys. A15 (2000), 5087.

[40] A.I. Davydychev, J. Phys. A25 (1992), 5587.

[41] N.I. Usyukina \& A.I. Davydychev, Phys. Atom. Nucl. 56 (1993), 1553.

[42] N.I. Usyukina \& A.I. Davydychev, Phys. Lett. B332 (1994), 159.

[43] N.I. Usyukina \& A.I. Davydychev, Phys. Lett. B348 (1995), 503.

[44] C. Studerus, Comput. Phys. Commun. 181 (2010), 1293.

[45] S.A. Larin \& J.A.M. Vermaseren, Phys. Lett. B303 (1993), 334.

[46] D.J. Broadhurst \& A.G. Grozin, Phys. Rev. D52 (1995), 4082.

[47] A.D. Kennedy, J. Math. Phys. 22 (1981), 1330.

[48] A. Bondi, G. Curci, G. Paffuti \& P. Rossi, Ann. Phys. 199 (1990), 268.

[49] A.N. Vasil'ev, S.É. Derkachov \& N.A. Kivel, Theor. Math. Phys. 103 (1995), 487. 
[50] A.N. Vasil'ev, M.I. Vyazovskii, S.É. Derkachov \& N.A. Kivel, Theor. Math. Phys. 107 (1996), 441.

[51] A.N. Vasil'ev, M.I. Vyazovskii, S.É. Derkachov \& N.A. Kivel, Theor. Math. Phys. 107 (1996), 710 . 